\title{
Über die Verwendung von Quecksilberoxyd bei der Analyse.
}

Von

\author{
Edgar F. Smith und Patl Heyl.
}

Berzeuius erwähnt in seinem Lehrbuch ${ }^{1}$ die Verwendung von Quecksilberoxyd zur Fällung von Zink, Nickel und Kobalt, und VolHARD ${ }^{2}$ empfiehlt, dals Zinksulfid, wie es im gewöhnlichen Lauf der Analyse erhalten wird, in Salzsäure aufgelöst und dann, nach genügender Konzentrierung dieser Lösung, Quecksilberoxyd zugesetzt und das Verdampfen bis zur Trockne fortgesetzt werde. Durch Erhitzen des Rückstandes wird Quecksilberchlorid ausgetrieben und Zinkoxyd bleibt zurück. Dieses Verfahren hat bedeutende Vorteile. Es kürzt den gewöhnlichen Lauf der Analyse ab und vermeidet Fehlerquellen, welche möglicherweise durch das Niederschlagen des Zinks vermittelst kohlensaurer Alkalien entstehen könnten. VOLHARD erwähnt auch, dals die Methode vielleicht mit Vorteil auf andere Sulfide angewandt werden könne. Es ist dies ein Punkt, welchen wir zu lösen versuchten, und im nachfolgenden geben wir daher die Einzelheiten unserer Beobachtungen.

Das bei den Versuchen gebrauchte Quecksilberoxyd war nach der Vorschrift von VoLHARD ${ }^{3}$ dargestellt.

Zink. Wir lösten zunächst nicht nur Schwefelzink, sondern auch gewogene Mengen reinen Zinkoxyds in Salzsäure auf und fügten, nach Konzentrierung der Flüssigkeit auf ein geringes Volumen, Quecksilberoxyd in genügendem Überschuls hinzu, um alle Flüssigkeit aufzunehmen, und verteilten es auch über die feuchten Wandungen des Tiegels. Die Verdampfung wurde dann bis zur Trockne fortgesetzt und der Rückstand 10 Minuten lang der Hitze einer gewöhnlichen Eisenplatte ausgesetzt, worauf er unter dem Rauchfang stark erhitzt wurde. Wir benutzten zu diesem Zwecke einen Platintiegel. Die Resultate waren wie folgt:

\footnotetext{
$110,139,145$.

${ }^{2}$ Lieb. Ann. 198, 331, 332.

${ }^{3} \mathrm{l}$. c.
} 


$\begin{array}{cc}\text { Angewandtes } \mathrm{ZnO}: & \text { Gefundenes } \mathrm{ZnO}: \\ 0.2180 \mathrm{~g} & 0.2179 \mathrm{~g} \\ 0.2155 \mathrm{~g} & 0.2156 \mathrm{~g} \\ 0.1437 \mathrm{~g} & 0.1441 \mathrm{~g} .\end{array}$

Cadmium. Um Cadmium quantitativ zu bestimmen, wird es entweder als Sulfid oder als Oxyd gewogen. Beim ersteren Verfahren ist ein grofser Zeitverbrauch unvermeidlich, und beim zweiten ist immer Gefahr vorhanden, durch Verflüchtigung Verluste herbeizuführen. Die elektrolytische Methode allein liefert zwar die besten Resultate, wird jedoch nicht häufig angewandt. Volumetrische Bestimmungsmethoden sind schwierig in der Ausführung. Es schien daher geraten, die Überführung des Chlorids in Oxyd vermittelst Quecksilberoxyd zu versuchen. Zu diesem Zwecke wurden gewogene Mengen reinen Cadmiumoxyds vermittelst Salzsäure in Porzellantiegeln gelöst, die Lösungen auf ein kleines Volumen eingedampft, und ein Überschuls von Quecksilberoxyd auf den Wandungen des Tiegels verteilt. Hierauf folgte das Verdampfen zur Trockne und Erhitzen:

$\begin{array}{cc}\text { Vorbandenes CdO: } & \text { Gefundenes CdO: } \\ 0.3467 \mathrm{~g} & 0.3435 \mathrm{~g}\end{array}$

Der Unterschied war offenbar der Flüchtigkeit von Cadmiumchlorid zuzuschreiben. Bei der nächsten Bestimmung trugen wir Sorge, die Seiten des Tiegels ganz dicht mit Quecksilberoxyd zu belegen, und die Mischung vor dem endlichen Erhitzen auf einer Ofenplatte zu trocknen.

Angewandtes $\mathrm{CdO}: \quad$ Gefundenes $\mathrm{CdO}$ :

$0.1233 \mathrm{~g}$ $0.1231 \mathrm{~g}$

Statt des Porzellantiegels benutzten wir dann einen Platintiegel und bestimmten den Betrag an Cadmium in einer salzsauren Lösung desselben von bekanntem Gehalt.

$\begin{array}{cc}\text { Angewandtes Cadmium: } & \text { Gefundenes Cadmium: } \\ 0.1522 \mathrm{~g} & 0.1516 \mathrm{~g} \\ 0.1218 \mathrm{~g} & 0.1224 \mathrm{~g}\end{array}$

Max Musprate ${ }^{1}$ versuchte, diese Methode auf Cadmium anzuwenden. Er bemerkt darüber wie folgt: ,Während die Methode für Zink befriedigend ist, hat sie sich für Cadmium als unnütz erwiesen $x \times \times \times$ Chlorcadmium ist so flüchtig, dafs es mit dem Quecksilberchlorid entweicht und die Resultate fallen zu niedrig aus." Es unterliegt keinem Zweifel, dals der Gebrauch ron Queck-

1 Journ. Soc. Chem. Ind. 13, 211. 
silberoxyd bei Cadmium mit gröfseren Schwierigkeiten verbunden ist, als bei irgend einem der von uns untersuchten Metalle. Wenn jedoch die folgenden Vorsichtsmaísregeln genau eingehalten werden, so hat man nicht den geringsten Verlust durch die Verflüchtigung ron Chlorcadmium zu befürchten. Man bediene sich eines Platintiegels. Ein Porzellantiegel hält bedeutende Mengen von Chlorcadmium an seinen Wandungen fest, während die Lösung eingedampft wird. Bei Verwendung eines Plantintiegels dagegen zieht sich kaum etwas an den Wandungen des Gefälses hinauf, sondern die ganze Menge von Chlorcadmium sammelt sich auf dem Boden des Tiegels an. Man verdampfe die Chlorcadmiumlösung zur Trockne, gleichgültig, ob sie freie Säure enthält oder nicht, und spritze die Wandungen des Tiegels mit gerade genug Wasser ab, um das Chlorcadmium zu lösen. Auch wende man einen genügenden Überschufs von Quecksilberoxyd an, um die ganze Lösung aufzunehmen und eine dicke Paste zu bilden, und streue das Oxyd gegen die feuchten Wandungen des Tiegels. Dann trockne man auf einer Ofenplatte und erhitze daselbst etwa 10 Minuten lang, bis Dämpfe von Quecksilberchlorid, welche durch den Überschufs von Oxyd zurückgehalten wurden, zu entweichen beginnen. Dann erhitze man zunächst bei sehr mäfsiger Wärme und lasse den Tiegel niemals schwach rotm glühend werden, bis alle Quecksilberchloriddämpfe entwichen sind. Hierauf mag volle Rotglühhitze angewandt werden, da das Cadmiumoxyd dadurch nicht affiziert wird.

Mangan. Bei der Analyse wird Mangan gewöhnlich als Schwefelverbindung erhalten und meistens als Manganomanganioxyd gewogen. Die Überführung des Schwefelmangans in Oxyd kann leicht und genau durch Verwendung von Quecksilberoxyd ausgeführt werden, so dafs der Gebrauch von Alkalien und das anhaltende Waschen der Manganverbindung wegfällt, welche nur zu häufig noch Unreinlichkeiten zurückhält.

Die Versuche mit Mangan wurden alle in Platingefälsen ausgeführt.

Eine Lösung von Manganosulfat lieferte nach Fällen mit kohlensaurem Natron per $25 \mathrm{ccm} 0.2109 \mathrm{~g} \mathrm{Mn}_{3} \mathrm{O}_{4}$. Andere Teile der Lösung wurden vermittelst Schwefelammonium niedergeschlagen und das erhaltene Schwefelmangan in Salzsäure gelöst. Die salzsaure Lösung wurde zur Trockne verdampft, um den Überschufs an Säure $\mathrm{zu}$ vertreiben, und der Rückstand in möglichst wenig Wasser gelöst. Sodann wurden die Tiegelwandungen wie oben mit einem 
Überschufs von Quecksilberoxyd möglichst vollständig bedeckt. Es hat sich herausgestellt, dafs diese Vorsichtsmalsregel unbedingt notwendig ist bei Metallen, deren Chlorverbindungen so flüchtig sind, dafs der an den Wandungen des Tiegels haftende Teil der Einwirkung des auf dem Boden des Gefälses befindlichen Quecksilberoxyds entgehen könnte, wodurch auf diese Weise Verlust eintritt. Cadmium ist ein Metall dieser Art.

Nach Hinzufügung des Quecksilberoxyds in der angegebenen Weise ist es nur notwendig, streng an den bei der Behandlung von Cadmium angegebenenVorschriftsmafsregeln festzuhalten. Es empfiehlt sich, das Manganoxyd bei voller Rotglut gut umzurühren.

Die nach dieser Methode erhaltenen Mengen von Manganoxyduloxyd waren per $25 \mathrm{ccm}$ obiger $\mathrm{MnSO}_{4}$-Lösung:

$$
\begin{aligned}
\text { I. }-0.2106 \mathrm{~g} & \text { III. }-0.2109 \mathrm{~g} \\
\text { II. }-0.2100 \mathrm{~g} & \text { IV. }-0.2112 \mathrm{~g}
\end{aligned}
$$

Nickel. Der Gehalt einer salzsauren Nickellösung wurde so eingerichtet, dals dieselbe in $25 \mathrm{ccm} 0.1269 \mathrm{~g} \mathrm{NiO}$ enthielt. Mehrere Teile dieser Lösung wurden, nach Hinzugabe ron Quecksilberoxyd, verdampft und das Nickeloxyd darin gefunden wie folgt:

$$
\begin{array}{ll}
\text { I. }-0.1443 \mathrm{~g} & \text { II. }-0.1444 \mathrm{~g}
\end{array}
$$

Eine wiederholte Bestimmung des Nickeloxyds in der ursprünglichen Lösung, durch Fällen mit Alkali, zeigte, dals das erste Resultat unrichtig war. In $10 \mathrm{ccm}$ der Lösung wurden durch Niederschlag mit Sodalösung $0.0579 \mathrm{~g} \mathrm{NiO}$ gefunden, während die Quecksilberoxydmethode $\mathrm{I}-0.0577 \mathrm{~g}$ und II $-0.0578 \mathrm{~g}$ gab. Diese Versuche mit Nickel wurden in Platingefälsen ausgeführt.

Das Schwefelnickel, wie es im Laufe der Analyse gefunden wird, mufs, nach der Lösung in Königswasser, zur Trockne verdampft, der Rückstand in möglichst wenig Wasser gelöst und dann gerade so behandelt werden wie oben angegeben ist.

Kobalt. Die Überführung von salzsaurem Kobalt in Oxyd durch Quecksilberoxyd ist eine vollständige, jedoch hat dieses Verfahren nur geringen praktischen Wert, weil es aufserordentlich schwierig ist, ein Kobaltoxyd von genügend konstanter Zusammensetzung für die endliche Wägung zu erhalten. Das Kobaltoxyd, welches wir aus einer Lösung von bekannter Stärke, durch Niederschlagen mit Alkali, erhielten, wog $0.1860 \mathrm{~g}$, während sein Gewicht bei Verwendung von Quecksilberoxyd I $0.1787 \mathrm{~g}$ und II $0.1803 \mathrm{~g}$ betrug. Das Metall wurde als Oxyd $\left(\mathrm{Co}_{3} \mathrm{O}_{4}\right)$ gewogen, da wir annahmen, dafs unter dem oxydierenden Einflufs des erhitzten Queck- 
silberoxyds seine Überführung in diese Form vollständig genug sein würde, um eine Wägung zu erlauben. Die bei der Quecksilberoxydmethode erhaltenen Oxyde sind jedoch in keiner Weise konstanter in Zusammensetzung als die bei der Verwendung von Alkali erhaltenen.

Da wir dachten, dal's möglicherweise das zweite Resultat $(I I=0.1803 \mathrm{~g}$ ) wegen unvollständigen Austreibens des Quecksilbers zu hoch sein möchte, so wurde der Rückstand nochmals bei voller Rotglühhitze erwärmt; er verlor dabei an Gewicht und wog $0.1761 \mathrm{~g}$. Bei nochmaligem Glühen (15 Minuten) bis auf schwache Rotglut nahm das Gewicht bis auf $0.1786 \mathrm{~g} \mathrm{zu}$.

Da diese Zahl dem Resultate von I $(0.1787 \mathrm{~g})$ sehr nahe kam, vermuteten wir, dal's möglicherweise bei niedriger Rotglut ein bestimmtes wägbares Oxyd entstehe, und daher wurden diese Oxyde, welche nach der Quecksilberoxydmethode hergestellt waren, soweit als möglich aus dem Tiegel entfernt, gewogen und im Wasserstoffstrom erhitzt mit folgenden Resultaten:

$$
\begin{gathered}
\text { Angewandtes Oxyd: } \\
\text { I. }-0.1259 \mathrm{~g} \\
\text { II. }-0.0630 \mathrm{~g}
\end{gathered}
$$

Berechnet für Kobalt in I:

$$
\begin{array}{ll}
\mathrm{CoO} & 0.0990 \mathrm{~g} \\
\mathrm{Co}_{3} \mathrm{O}_{4} & 0.0928 \mathrm{~g} \\
\mathrm{Co}_{2} \mathrm{O}_{3} & 0.0895 \mathrm{~g}
\end{array}
$$

Gefundenes Kobalt:

$$
\text { I. }-0.0916 \mathrm{~g}
$$$$
\text { II. }-0.0457 \mathrm{~g}
$$

Berechnet für Kobalt in II:

$0.0496 \mathrm{~g}$

$0.0465 \mathrm{~g}$

$0.0448 \mathrm{~g}$

Eine Prüfung dieser Zahlen zeigt, dafs die Menge des gefundenen Kobalts in beiden Fällen zwischen den für die Oxyde $\mathrm{Co}_{3} \mathrm{O}_{4}$ und $\mathrm{Co}_{2} \mathrm{O}_{3}$ theoretisch erforderlichen Mengen lag. Die Oxydmenge beim II. Versuch war zu klein, um eine zuverlässige Formel hieraus abzuleiten. Das Oxyd, welches einen dem in No. I gefundenen nächstkommenden Kobaltgehalt hat, ist $\mathrm{Co}_{3} \mathrm{O}_{4}$; es verlangt das Verhältnis $\mathrm{C}_{0}: \mathrm{O}=1: 1.333$. Dieses Verhältnis ergab sich bei diesem Versuch wie 1:1.4. Im ganzen kann man sich also auf die Oxyde von Kobalt nicht verlassen, da verschiedene Temperaturbedingungen die Bildung verschiedener Oxyde veranlassen. Es mag möglich sein, bei niedriger Rotglut ein Oxyd von annähernd der Zusammensetzung $\mathrm{CO}_{3} \mathrm{O}_{4}$ darzustellen, es besteht jedoch keine Sicherheit in dieser Beziehung. Es ist daher am besten, die Oxyde zu Metall zu reduzieren und in dieser Form zu wägen.

Wismut. Bei Trennungen wird dieses Metall häufig als Oxychlorid niedergeschlagen. Seine Überführung durch Quecksilberoxyd 
ist von bedeutender Wichtigkeit in analytischer Beziehung. Wir versuchten zunächst, das Oxychlorid zu transponieren, indem wir es in Wasser mit Quecksilberoxyd zusammenbrachten, dann verdampften und den Rückstand erhitzten; jedoch sahen wir uns gezwungen, dieses Verfahren aufzugeben und lösten das niedergeschlagene Oxychlorid in einigen Tropfen Salzsäure, fügten genügend Quecksilberoxyd hinzu, um die Säure zu neutralisieren, und aufserdem einen für die Reaktion genügenden Uberschuls. Nach dem Verdampfen wurde wie beim Mangan verfahren. Sollte der Rückstand gelegentlich hier und da schwarze Flecken zeigen, so befeuchte man ihn mit Wasser und 1-2 Tropfen Salpetersäure. Nach dem Eintrocknen und abermaligem Glühen bei voller Rotglühhitze wäge man als Wismuttrioxyd. Bei dieser Operation sollen Porzellantiegel angewandt werden.

Resultate:

Gefundenes Wismut: $0.0941 \mathrm{~g}$ $0.0759 \mathrm{~g}$
Angewandtes Wismut:

$0.0945 \mathrm{~g}$ $0.0756 \mathrm{~g}$

Da wir die obigen Methoden wiederholt mit zufriedenstellenden Resultaten anwandten, so möchten wir dieselben empfehlen, da sie unserer Meinung nach den Zeitfaktor bedeutend verringern, und weil aufserdem durch die Einführung von reinem Quecksilberoxyd die Gelegenheit zur Beimischung von Unreinlichkeiten durch gewöhnliche Reagentien wesentlich verringert ist. Unter den Studierenden am hiesigen Laboratorium ist es eine gewöhnliche Operation, das Schwefelzink, wie es bei der Analyse von Zinkblende oder kieselsaurem Zink erhalten wird, in oben beschriebener Weise in Oxyd überzuführen, und die Resultate waren stets zufriedenstellend.

Persoz und Berzeurus benutzten Quecksilberoxyd zu Fällungen bei der Trennung von Metallen, und in der früher erwähnten Mitteilung von VouHard erwähnt der Verfasser, dafs es ihm gelungen sei, Eisenoxyd von Mangan vermittelst Quecksilberoxyd zu trennen. Er hat jedoch keine Resultate angegeben, und wir haben uns daher erlaubt, diese Arbeit zu wiederholen. Es wurde eine Lösung von Eisenchlorid hergestellt durch Auflösen von Klavierdraht in Königswasser; $25 \mathrm{ccm}$ dieser Lösung enthielten $0.1359 \mathrm{~g} \mathrm{Fe.} \mathrm{Zu} 25 \mathrm{ccm}$ der $\mathrm{FeCl}_{3}$-Lösung fügten wir $0.3 \mathrm{~g}$ Manganchlorür. Quecksilberoxyd wurde zu der kalten Mischung gegeben, das Eisenoxydhydrat sofort vermittelst der Saugpumpe abfiltriert, mit kaltem Wasser gewaschen, in Salzsäure gelöst und mit Quecksilberoxyd wieder niedergeschlagen. 
Der zweite Niederschlag wurde sorgfältig gewaschen und dann in einem gewogenen Platintiegel geglüht.

VoLHard giebt keine anderen Anweisungen in Bezug auf diese Trennung, als die folgenden: „Die verdünnte, neutrale Lösung der Chloride wurde mit Quecksilberoxyd behandelt." Wir verfahren bei unserer Arbeit auf folgende Weise: Man füge einen Uberschuls des Fällungsmittels zu der verdünnten Lösung der gemischten Chloride, welche sich in einer EruendEYERschen Flasche befinden. Eine Spur freier Säure ist in der Flüssigkeit vorhanden. Man schüttele die Flasche und füge, wenn, nach dem Absitzen des Niederschlages, die darüberstehende Flüssigkeit eine rötliche oder gelbe Färbung zeigt, mehr Quecksilberoxyd hinzu und fahre so fort, bis die Flüssigkeit farblos ist. Nach Auflösen des ersten Eisenniederschlages auf dem Filter in Salzsäure füge man allmählich Ammoniak hinzu, bis nahezu alle freie Säure neutralisiert ist, und wiederhole das Niederschlagen. Die von uns erhaltenen Filtrate erwiesen sich frei von Eisen.

$\begin{array}{ccc}\text { Angew. Eisen: } & \text { Gef. Eisen: } & \text { Gleichzeitig vorh. } \mathrm{MnCl}_{2} \text { : } \\ 0.1359 \mathrm{~g} & 0.1363 \mathrm{~g} & 0.3 \mathrm{~g} \\ - & 0.1356 \mathrm{~g} & -\end{array}$

Die Totalverdünnung bei diesen Bestimmungen betrug $150 \mathrm{ccm}$.

Es ist möglich, das Eisen, frei von Mangan und fertig zum Glühen, in 45 Minuten zu isolieren.

Bei einem Versuche, Berylliumoxyd in der gerade beschriebenen Weise zu fällen, fanden wir, dals, selbst unter den verschiedensten Bedingungen, das Filtrat stets jenes Element noch enthielt. Der Versuch daher, Eisen von Beryllerde zu trennen, erwies sich als erfolglos.

Uran wird aus seinen Chloridlösungen in der Kälte durch Quecksilberoxyd selbst bei 12stündiger Einwirkung nicht vollständig niedergeschlagen. Beim Verdampfen der Chloridlösung zur Trockne und Glühen blieb ein dunkelgrün gefärbtes $\mathrm{Oxyd}\left(\mathrm{U}_{3} \mathrm{O}_{4}\right)$ zurück. Da diese Umwandlung in Oxyd eine vollständige ist, so erschien es uns möglich, auf diese Weise eine Trennung des Urans von den Alkalien zu bewerkstelligen. Unsere Bemühungen in dieser Richtung waren jedoch nicht erfolgreich. Das Nichtausfallen des Urans aus salzsaurer Lösung veranlafste uns, die Trennung des Eisens von Uran in derselben Weise wie diejenige des Eisens von Mangan zu versuchen, die Resultate waren aber besonders unzufriedenstellend, da bis zu $85 \%$ Uran mit dem Eisenoxyd niedergeschlagen wurden. 
Die Trennung des Eisens von Nickel, welche auf dieselbe Weise wie mit Mangan ausgeführt wurde, gab ein annäherndes Resultat. Nickel ist anscheinend weniger reaktiv mit Quecksilberoxyd als Uran.

Gef. Eisen:

Angew. Eisen:

Vorhandenes Nickel:

$0.1372 \mathrm{~g}$

$0.1359 \mathrm{~g}$

$0.1000 \mathrm{~g}$

Wenn vornehmlich Schnelligkeit der Analyse ohne grofse Genauigkeit allein verlangt wird, so mag diese Art der Trennung als zufriedenstellend betrachtet werden.

Die Trennung des Eisens von Nickel und Kobalt wurde auf dieselbe, soeben mitgeteilte Weise ausgeführt. Die Resultate besafsen ungefähr denselben Grad von Genauigkeit.

VoLHARD erwähnt auch, dals Aluminium aus kalten, salzsauren Lösungen durch Queksilberoxyd niedergeschlagen wird. Wir stellten deshalb eine Aluminiumlösung her, welche Chlorberyllium enthielt. Beim Behandeln mit Quecksilberoxyd, wie bei der Trennung des Eisens von Aluminium angegeben, bemerkten wir, dal's einerseits das Aluminium nieht vollständig niedergeschlagen wurde, und andererseits sehr beträchtliche Mengen von Berylloxyd mit dem Thorerdehydrat zusammen ausgefallen waren.

Behandelt man salzsaures Lanthan in der Kälte mit Quecksilberoxyd, so fällt $1 / 5$ des Lanthans als Hydroxyd aus; in heifsen Lösungen wurden etwa $75 \%$ des gesamten Lanthans niedergeschlagen. Das Reagenz zeigte nahezu dieselben Resultate bei Behandlung von heilsen oder kalten salzsauren Lösungen von Cerium.

Von Berzeurus ${ }^{1}$ wird erwähnt, dals Zink von Mangan getrennt werden kann, indem man Quecksilberoxyd za der salzsauren Lösung beider Metalle giebt. Unsere Erfahrung in Bezug auf salzsaure Zinklösungen und Quecksilberoxyd ist kurz folgende: Die Fällung ist nur eine teilweise in kalten Lösungen; Hitze beschleunigt dieselbe und das Quecksilberoxyd wird anfangs deutlich weifs und dann schwarz. In einem Falle, da $0.2176 \mathrm{~g}$ Zinkoxyd vorhanden war, erhielten wir $0.0288 \mathrm{~g}$. Dieser Versuch wurde jedoch in der Kälte ausgeführt. Bei einem zweiten Versuch und einer Temperatur von etwa $80^{\circ} \mathrm{C}$. fanden wir statt $0.2176 \mathrm{~g}$ Zinkoxyd $0.2047 \mathrm{~g}$. Wir können nicht einsehen, wie dieser Prozels eine Trennung des Zinks von Mangan möglich machen kann, da letzteres ebenfalls durch Quecksilberoxyd aus warmen Lösungen seiner Salze ausgeschieden wird.

1 Lehrb. d. Chem. 10, 145. 
Ein Versuch, Chrom von den Alkalien auf diese Weise zu trennen, gab so unzufriedenstellende Resultate, dafs die Einzelheiten hier weggelassen werden.

Fassen wir nun die Resultate in Bezug auf den Gebrauch von Quecksilberoxyd als Fällungsmittel zusammen, so lälst sich Eisen, Chrom und Aluminium (VoxHARD) vollständig aus kalten Lösungen niederschlagen, während Zink, Kobalt, Nickel, Uran, Beryllium, Cerium und Lanthan nur teilweise ausgefällt werden; ihre Fällung ist in heifsen Lösungen vollständiger. $\mathrm{Da}$ in kalten Lösungen Spuren von Mangan niedergeschlagen werden können, so ist es immer rätlich, das Eisen doppelt zu fällen, um die Trennung von Mangan zu einer vollständigen und befriedigenden $\mathrm{zu}$ gestalten. ${ }^{1}$

1 Im Anschluls an obige ausführlichen Mitteilungen über die Verwendungen des Quecksilberoxydes in der Analyse sei der Hinweis gestattet, dals sich in den aus dem Nachlasse von Clemens Zimmermann durch Georg Alibegoff und Gerhard Krứss herausgegebenen Abhandlungen: „Untersuchungen über das Uran“, Lieb. Ann. 232, 273-324, und „Über die Atomgewichte des Kobalts und Nickels" Lieb. Ann. 232, 324-347, ebenfalls eine Anzahl von wichtigen Beobachtungen über die analytische Verwendung des Quecksilberoxydes vorfinden.

G. KRï̀ss.

Universität von Pennsylvanien, im Juni 1894.

Bei der Redaktion eingegangen am 1. Juli 1894. 